\title{
Rapid Measurements of Intensities for Safety Assessment of Advanced Imaging Sequences
}

Jensen, Jørgen Arendt; Rasmussen, Morten Fischer; Stuart, Matthias Bo; Tomov, Borislav Gueorguiev

Published in:

Proceedings of the SPIE - Progress in Biomedical Optics and Imaging

Link to article, DOI:

$10.1117 / 12.2043688$

Publication date:

2014

Document Version

Early version, also known as pre-print

Link back to DTU Orbit

Citation (APA):

Jensen, J. A., Rasmussen, M. F., Stuart, M. B., \& Tomov, B. G. (2014). Rapid Measurements of Intensities for Safety Assessment of Advanced Imaging Sequences. In Proceedings of the SPIE - Progress in Biomedical Optics and Imaging (Vol. 9040). [90400Z] SPIE - International Society for Optical Engineering. https://doi.org/10.1117/12.2043688

\section{General rights}

Copyright and moral rights for the publications made accessible in the public portal are retained by the authors and/or other copyright owners and it is a condition of accessing publications that users recognise and abide by the legal requirements associated with these rights.

- Users may download and print one copy of any publication from the public portal for the purpose of private study or research.

- You may not further distribute the material or use it for any profit-making activity or commercial gain

- You may freely distribute the URL identifying the publication in the public portal 
Paper presented at the SPIE Medical Imaging conference, San Diego, USA, 2014:

\section{Rapid Measurements of Intensities for Safety Assessment of Advanced Imaging Sequences}

Jørgen Arendt Jensen, Morten Fischer Rasmussen, Matthias Bo Stuart and Borislav G. Tomov

Center for Fast Ultrasound Imaging, Biomedical Engineering group, Department of Electrical Engineering, Bldg. 349, Technical University of Denmark, DK-2800 Kgs. Lyngby, Denmark 


\title{
Rapid Measurements of Intensities for Safety Assessment of Advanced Imaging Sequences
}

\author{
Jørgen Arendt Jensen, Morten Fischer Rasmussen, Matthias Bo Stuart and Borislav G. Tomov \\ Center for Fast Ultrasound Imaging, Department of Electrical Engineering, \\ Technical University of Denmark, DK-2800 Lyngby, Denmark
}

\begin{abstract}
FDA requires that intensity and safety parameters are measured for all imaging schemes for clinical imaging. This is often cumbersome, since the scan sequence has to broken apart, measurements conducted for the individually emitted beams, and the final intensity levels calculated by combining the intensities from the individual beams. This paper suggests a fast measurement scheme using the multi-line sampling capability of modern scanners and research systems. The hydrophone is connected to one sampling channel in the research system, and the intensity is measured for all imaging lines in one emission sequence. This makes it possible to map out the pressure field and hence intensity level for all imaging lines in a single measurement. The approach has several advantages: the scanner does not have to be re-programmed and can use the scan sequence without modification. The measurements are orders of magnitude faster (minutes rather than hours) and the final intensity level calculation can be made generic and reused for any kind of scan sequence by just knowing the number of imaging lines and the pulse repetition time. The scheme has been implemented on the Acoustic Intensity Measurement System AIMS III (Onda, Sunnyvale, California, USA). The research scanner SARUS is used for the experiments, where one of the channels is used for the hydrophone signal. A $3 \mathrm{MHz}$ BK 8820e (BK Medical, Herlev, Denmark) convex array with 192 elements is used along with an Onda HFL-0400 hydrophone connected to a AH-2010 pre-amplifier (Onda Corporation, Sunnyvale, USA). A single emission sequence is employed for testing and calibrating the approach. The measurements using the AIMS III and SARUS systems after calibration agree within a relative standard deviation of $0.24 \%$. A duplex B-mode and flow sequence is also investigated. The complex intensity map is measured and the time averaged spatial peak intensity is found. A single point measurement takes 3.43 seconds and the whole sequence can be characterized on the acoustical axis in around 6 minutes.
\end{abstract}

\section{INTRODUCTION}

All imaging mode in medical ultrasound scanners has to abide by FDA rules for intensity levels and safety limits. ${ }^{1}$ This necessitates the measurements of the emitted pressure of the transducer as a function of spatial position. Modern ultrasound scanners often employ very advanced imaging schemes, where emissions for B-mode imaging, blood flow estimation, and tissue motion estimation are interleaved. Each of these emissions are in different directions with different transmit foci and pulse lengths, which complicates the measurements. Water tank systems are employed, and they are often only capable of measuring one emission at a time in order to average the response to yield the best possible result. Often these systems are not synchronized to the scanner emitting the pulse and averaging has to be performed using special algorithms for aligning multiple measurements. Each measurement can only be conducted on one imaging line at a time, as the system cannot differentiate between imaging lines. A complicated sequence therefore has to be measured in several steps. First, the imaging sequence has to be divided into the individual emissions, and then the responses as a function of space are measured. This is a lengthy procedure, since the hydrophone has to be moved between individual steps, and the sampling has to be performed with a high density as the fields are narrowly focused. Often F-numbers from $2-5$ are found for the emitted field, and this gives a lateral Full-Width-Half-Max (FWHM) around $2-5 \lambda$ and a corresponding spatial sampling period 5-10 times lower than the FWHM. Covering a sufficient area for finding the spatial peak can then take hours as the movement and settling time is on the order of 1-2 seconds for the motion and averaging. This has to be performed for all emissions in a sequence, which can amount to several hundred.

For further author information send correspondence to Jørgen Arendt Jensen, E-mail: jaj@elektro.dtu.dk 
Methods for cutting down on this time can be devised. One common method is just to take the most dominant emission and then assume this is employed all the time. This will ensure a very conservative estimate of intensities, but it can also result in a maximum emitted energy much below the allowed limit. This results in a poorer penetration depth and less accurate velocity estimates. A combination of lines can then be used, but this still involves a judgment to make the selection that has to be conservative. A major error is also that the scanner has to be reprogrammed from the actual scanning sequence. This can, especially for experimental research systems, be cumbersome and error prone.

This paper suggests a method for solving these problems and minimizing the measurement time. Today multichannel sampling systems for experimental use have been developed like the RASMUS system, ${ }^{2}$ ULA-OP, ${ }^{3}$ and the experimental SARUS scanner. ${ }^{4}$ All modern scanners also offer internal real-time sampling of signals from the transducer. The approach suggested here is therefore to use these sampling capabilities to acquire the hydrophone signal. One sampling channel is then connected to the hydrophone, and the pressure level as a function of emission number is thereby recorded. This has several advantages. Foremost the scanner does not have to be reprogrammed, only the sampling of the hydrophone has to be added. It is therefore guaranteed that the actual imaging sequence is measured and validated. Secondly, the signal measured is in phase with the emitted pulse and can therefore directly be averaged without processing. The last and really major advantage is that the measurement is much faster. The sampling is performed in real-time, and emitted pressures for all imaging directions are acquired in one measurement. The complete measurement can be performed in seconds for several images, even using a moderate pulse repetition time between emissions to avoid reverberations and rereflections in the tank. The correct and full intensity profile for the image can then be derived and the complete intensity level easily calculated.

Normally the measurement should be performed for all depths and lateral positions to find the spatial peak values, but this can also be optimized. Often a scan sequence is symmetric and mostly concentrated at the center of the image. The spatial peak will therefore be on the center acoustical axis of the transducer and the levels need only be measured on this axis, thus, eliminating one dimension in the scan. A proper alignment of the transducer can easily be performed as described in Section 4, and the measurement performed on the acoustical axis. This can also be validated using field simulations to show that the peak intensities are found at the center for the image as described in Section 3.

The approach has been implemented on the SARUS experimental ultrasound scanner using a BK 8820e (BK Medical, Herlev, Denmark) transducer and the AIMS III intensity measurement system (Onda Corporation, Sunnyvale, California, USA) as described in Section 4. Results for the approach are described in Section 5 and commented on in Section 7. It is shown that intensity mapping can be conducted within 10 minutes rather than the hours or days currently needed for this, and it is argued that the approach is less error prone and more accurate than the more cumbersome and manual approaches. It can be implemented in any ultrasound scanner that has the possibility of connecting an external input to a single sampling channel in the scanner.

\section{FDA INTENSITY CALCULATION}

The safety limits imposed by FDA are derived from the acoustic pressure $p(t, \vec{r})$ measured in a water bath using a calibrated hydrophone. The instantaneous intensity is given by

$$
I_{i}(t, \vec{r})=\frac{p^{2}(t, \vec{r})}{\rho c}=\frac{p^{2}(t, \vec{r})}{Z} .
$$

where $Z=\rho c$ is the characteristic acoustic impedance, $\rho$ is the density, $t$ is time, $\vec{r}$ is the position of the measurement, and $c$ is the speed of sound. The field can be characterized by the spatial and temporal peak of $I_{i}$, denoted $I_{s p t p}$, or it can be averaged over time giving the spatial peak temporal averaged intensity

$$
I_{s p t a}=\frac{1}{T^{\prime}} \int_{0}^{T^{\prime}} I_{i}\left(t, \vec{r}_{\max }\right) d t
$$


where $T^{\prime}$ is the period from pulse to pulse and $\vec{r}_{\max }$ denotes the position of maximum intensity. The discrete version of the temporal average intensity from the sampled acoustic pressure $p(n, \vec{r})$ is:

$$
I_{t a}=\sum_{n=1}^{N} \frac{p^{2}(n, \vec{r})}{Z} \frac{\Delta T}{T_{p r f}}
$$

where $T_{p r f}$ is the time between pulse emissions, $\Delta T$ is the sampling interval, and $N$ is the number of samples in the response. It is assumed that the same emission is used continuously, but often different pulses in different direction are used. They should then be weighted according to their relative time duration in the imaging sequence as

$$
I_{t a}=\frac{1}{M} \sum_{i=1}^{M} \sum_{n=1}^{N} \frac{p_{i}^{2}(n, \vec{r})}{Z} \frac{\Delta T}{T_{p r f}(i)}
$$

where $i$ is the emission number and $M$ is the number of emissions in the complete sequence. A derated value can also found by employing an attenuation value of $0.3 \mathrm{~dB} /[\mathrm{MHz} \mathrm{cm}]$ on the pulse at the center frequency.

A second quantity is the Mechanical Index (MI) given by

$$
M I=\frac{\left|\min (p(n, \vec{r})) / 10^{6}\right|}{\sqrt{f_{0} / 10^{6}}}
$$

where $\min (p(n, \vec{r}))$ is the peak negative pressure and $f_{0}$ is the emitted frequency of the probe. These are the two quantities calculated in this study.

\section{SIMULATION TO FIND MEASUREMENT POSITIONS}

Before measuring the intensities, the spatial position of the highest intensity should be determined. This can be complicated for a complex imaging sequence as different foci are used for different emissions and each emission could include few or many elements and with different transmit apodizations. The time between emissions can also vary, and therefore the relative weight between emissions will vary. It is, therefore, in general complicated to determine the peak intensity position.

Mapping out the intensity for all positions will necessitate a large number of measurements, and this can be considerably reduced by simulating the intensity field prior to the measurements. The Field II program ${ }^{5,6} \mathrm{can}$ be used for calculating an intensity map and thereby determine the correct position. The simulation program is linear whereas non-linear fields are found in water tank measurements. This will change the values especially for high pressures, but the relative distribution of the intensities will be the same. The program can therefore be used for finding the region for the measurements, and they should then be conducted along the axis of highest intensity to capture the evolution in depth due to the non-linear effect.

\section{EXPERIMENTAL SETUP}

The experimental scanner SARUS 4 is used for emitting the sound and measuring the response. The system is connected to a $3 \mathrm{MHz}$ BK 8820e (BK Medical, Herlev, Denmark) convex array transducer with 192 transducer elements. The Acoustic Intensity Measurement System AIMS III (Onda, Sunnyvale, California, USA) is used for moving the Onda HFL-0400 (lipstick) hydrophone. A AH-2010 (Onda Corporation, Sunnyvale, USA) 20 $\mathrm{dB}$ amplifier is used coupled to the hydrophone after a ATH-2000 $20 \mathrm{~dB}$ attenuator to prevent overload in the measurement system.

Two imaging sequences have been used for the experiments. The first is a simple fixed focus sequence with a focus at $40 \mathrm{~mm}$, and the beam direction on the center axis of the transducer. This is used for calibrating the SARUS system and for comparison between the Onda and the SARUS system. The second sequence makes a simple duplex scan, where a 129 line B-mode sequence is interleaved with 129 flow emissions. Such a sequence is typically used in spectral velocity estimation. These two sequences give the intensity distributions for typical sequences used in modern scanners. The approach described here is, however, not limited to these modes as any other kind of mode can be used (CFM, VFI, synthetic aperture, plane wave, continuous wave, 3D, etc.). 


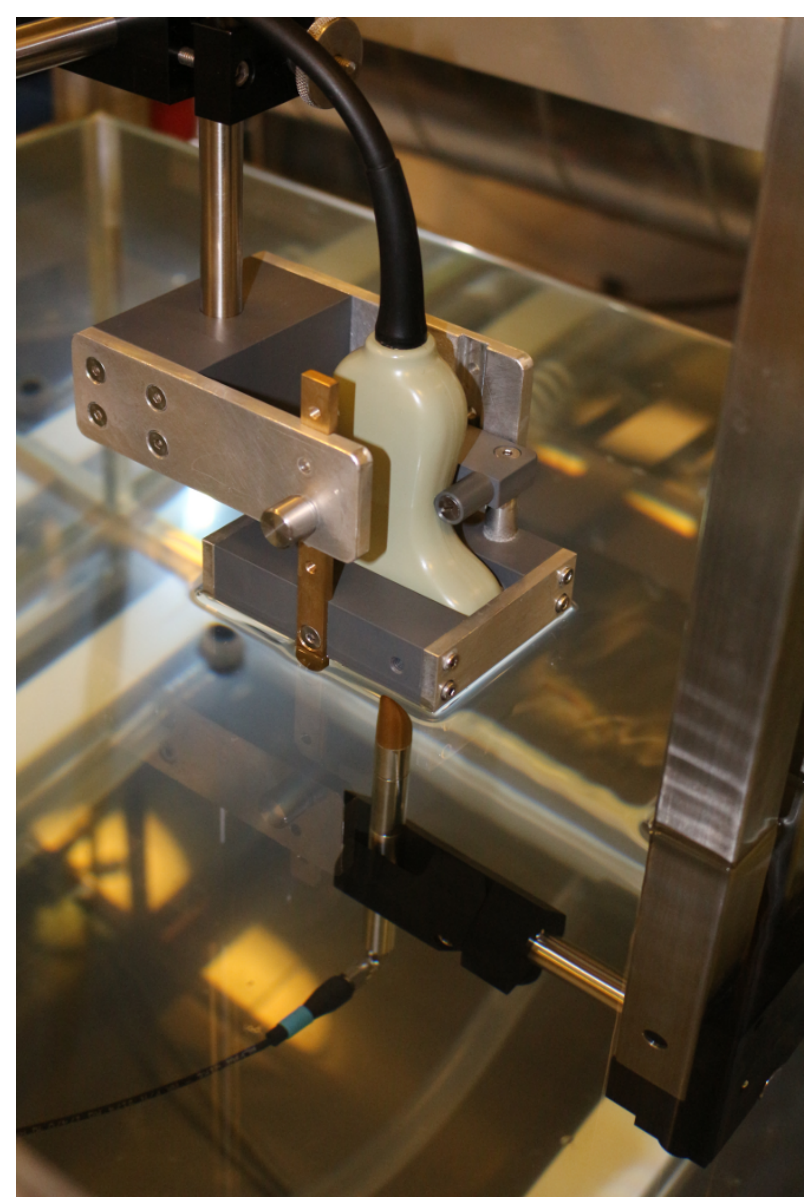

Figure 1. Set-up for the intensity measurement. The convex array transducer is fixed in the holder and the hydrophone mounted on the translation stage is moved relative to the probe.

During measurements the pulse repetition frequency is set artificially low to $100 \mathrm{~Hz}$ to avoid reverberations and multiple reflections in the tank. The hydrophone amplifier is connected to one receive channel on the SARUS system which has a maximum input range of \pm 1 volts. Both the measurement systems can be fully controlled using Matlab (Mathworks, MA, USA). The measurements can readily be averaged, since the emission and hydrophone measurement are performed by the same system and therefore are fully in phase. This makes it possible to average even complicated measurement sequences without phase errors, and it is, thus, not needcessary to resort to phase correction schemes used in other systems for getting usable averages.

The basic setup is shown in Fig. 1 with the mounting of the transducer on the fixed boom and the hydrophone mounted on the translation stage. The coordinate follow the usual convention of $z$ being the depth direction, $x$ along the transducer crystals, and $y$ in the elevation direction. The hydrophone and transducer are then aligned by finding the peak position of the pressure for a ultrasound field focused directly down with an F\# of 2 and using 64 elements in transmit. The hydrophone is moved across the beam in the $x$ direction to find a value before the maximum at $x_{m}-\Delta_{x}$ and at two positions after the maximum at $x_{m}$ and $x_{m}+\Delta_{x}$, where $\Delta_{x}$ is the step size. A second order polynomial is then fit to the energy of the response, and the position of the maximum value is found by the interpolation formula: ${ }^{7}$

$$
x_{i n t}=x_{m}-\Delta_{x} \frac{E\left(x_{m}+\Delta_{x}\right)-E\left(x_{m}-\Delta_{x}\right)}{\left.2\left(E\left(x_{m}+\Delta_{x}\right)-2 E\left(x_{m}\right)\right)+E\left(x_{m}-\Delta_{x}\right)\right)} .
$$

This gives a very accurate determination of the peak position without having to move a large number of steps. The procedure is repeated in the $y$ direction. The hydrophone is then placed before the focus and after the focus, 
and the $x-y$ procedure is repeated for both placements. From these two maximum energy value positions the unit vector for the coordinate system can be found along with the center position of the aperture. The motion can then be performed in the transducer coordinate system rather than the Onda coordinate system.

The basic setup in the measurement is therefore:

1. Set the system to emit a focused field along the acoustical axis of the transducer.

2. Manually align the hydrophone to be at the center axis of the transducer.

3. Use the automatic alignment program to find the unit vector for the acoustical axis along with the center position of the aperture.

4. Measure the imaging sequence a number of times, and store the data in Matlab files.

5. Repeat the image sequence measurement over the region of interest.

It should be ensured that the imaging sequence measured is the same as used for clinical scanning. This is ensured by setting up the sequence for a scan and acquire a single measurement corresponding to a clinical scan. The SARUS system is then programmed for the exact scan, and a second script is run that modifies the setup, so that the receiving channel is attached to the hydrophone. The script then runs the clinical emission sequence automatically, and thereby ensures that the correct sequence is executed by the scanner. The hydrophone then records the pressure waves for all the emissions at one spatial position in the time span for one clinical image.

\section{RESULTS}

This Section shows results from both comparing the Onda and SARUS measurements and for characterizing the duplex imaging sequence.

\subsection{Comparisons between systems}

The first measurement is for calibrating the system. The single emissions and focusing sequence are employed and both the Onda software and SARUS are used for measuring the response in two measurements. The pressure at the peak position is then used for finding the scaling factor for the SARUS system.

The pressure waveforms measured by both systems are shown in Fig. 2. The waveforms are measured on the acoustical axis of the probe at the position of peak pressure. The SARUS waveform is scaled by a factor of 1.367, so the peak values of the two waveforms coincide. Small differences are seen between the waveforms.

The hydrophone was then moved for depths from $20 \mathrm{~mm}$ to $120 \mathrm{~mm}$ along the acoustical axis of the field. The resulting $I_{t a}$ is shown in Fig. 3. The calibrated $I_{\text {spta }}$ for this sequence is $2.29 \mathrm{~mW} / \mathrm{cm}^{2}$ due to the low $f_{p r f}$ of $100 \mathrm{~Hz}$. The relative error between the AIMS III system and SARUS is $0.24 \%$ indicating a negligible difference between the measurements. Sampling using a single channel in the SARUS system can, thus, be used for finding correct intensity levels.

\subsection{Measurements on duplex sequence}

The second example is a duplex scan sequence, where flow and B-mode emissions are intermixed. This gives a complex distribution of energy in the medium under investigation. It is depicted in Fig. 4, where the instantaneous intensity is shown as a function of emission number and depth. The level is shown in $\mathrm{dB}$ relative to an instantaneous intensity of $1 \mathrm{~W} / \mathrm{cm}^{2}$ with a $60 \mathrm{~dB}$ dynamic range. The measurement is conducted at a depth of $42 \mathrm{~mm}$ with the hydrophone on the center axis of the transducer. This coincides with the transmit focus for the B-mode sequence.

The alternation between flow and B-mode emissions can be seen as every second emission is a four cycle transmission suitable for velocity estimation. The emitted B-mode field is seen as the butterfly shape at the center of the image, since the imaging beams are swept across the hydrophone position. The measurement is conducted at a pulse repetition frequency of $100 \mathrm{~Hz}$ to avoid re-reflections in the water tank. Assuming it to be $8 \mathrm{kHz}$ for the real measurement gives a value of $I_{t a}=245 \mathrm{~mW} / \mathrm{cm}^{2}$. 


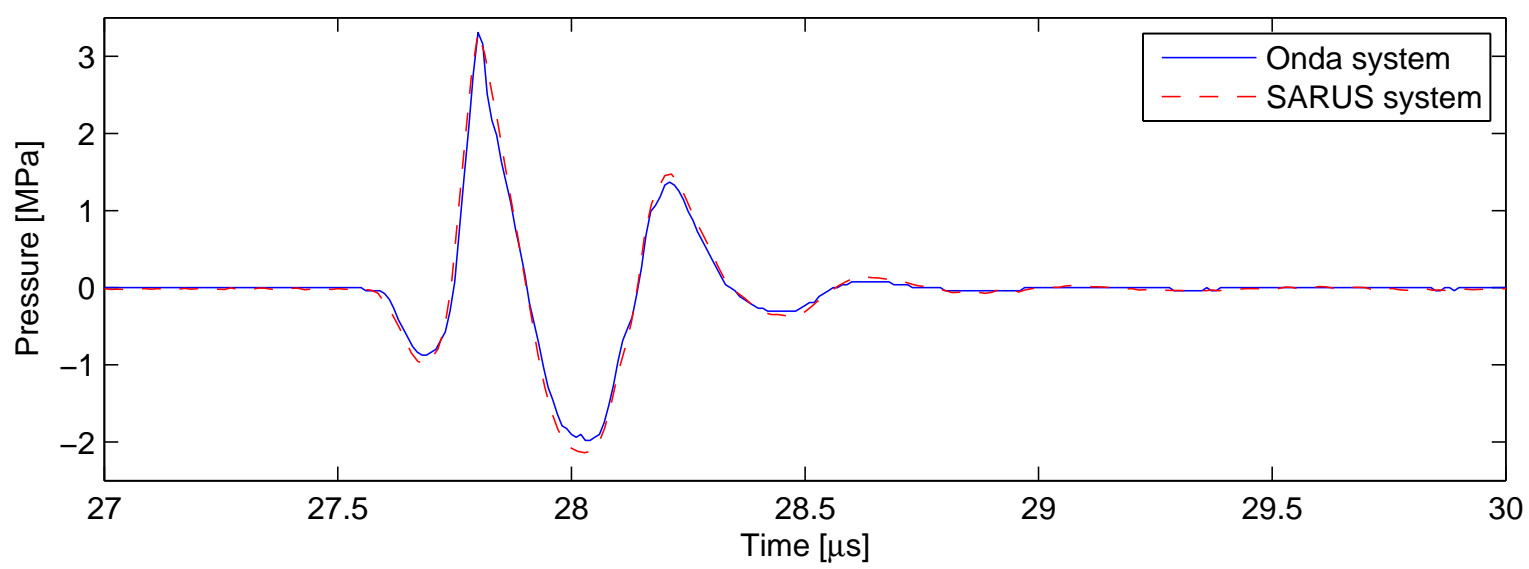

Figure 2. Pressure waveforms measured by the Onda system (blue) and the SARUS system (dashed red) at the peak pressure position for the single focus emission. The SARUS waveform is scaled by a factor 1.366 to yield the same peak pressure value as from the Onda system.

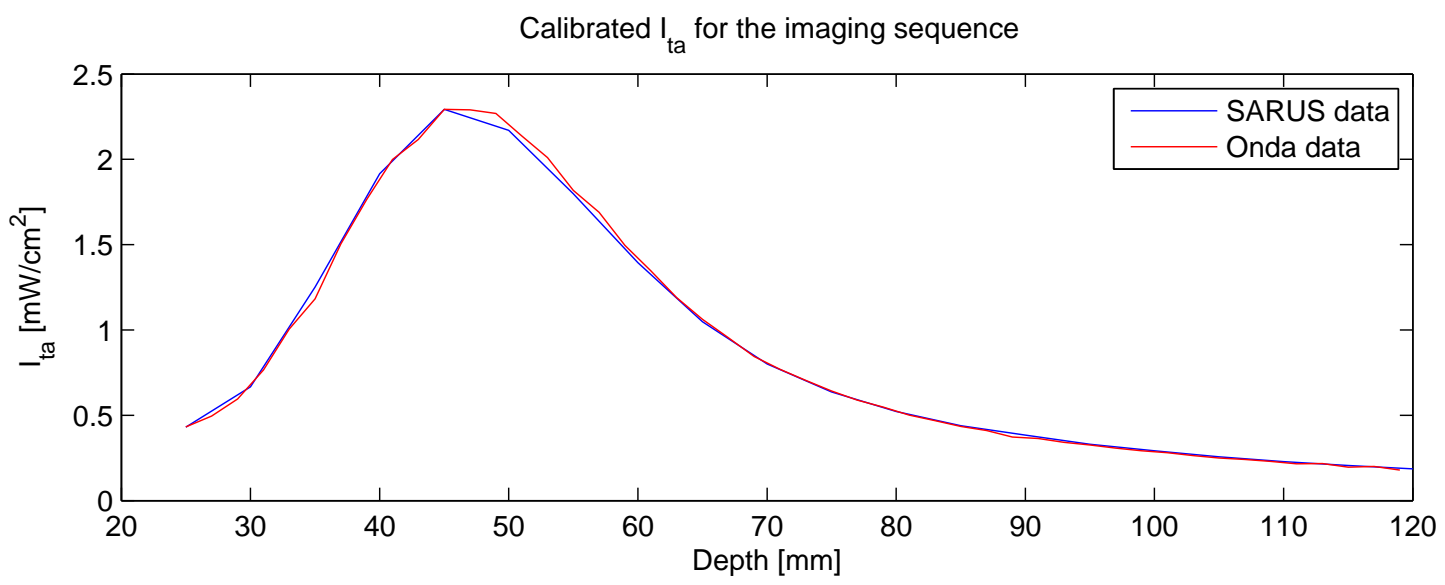

Figure 3. Calibrated value for $I_{t a}$ measured by the SARUS scanner compared to the values from the Onda system. 


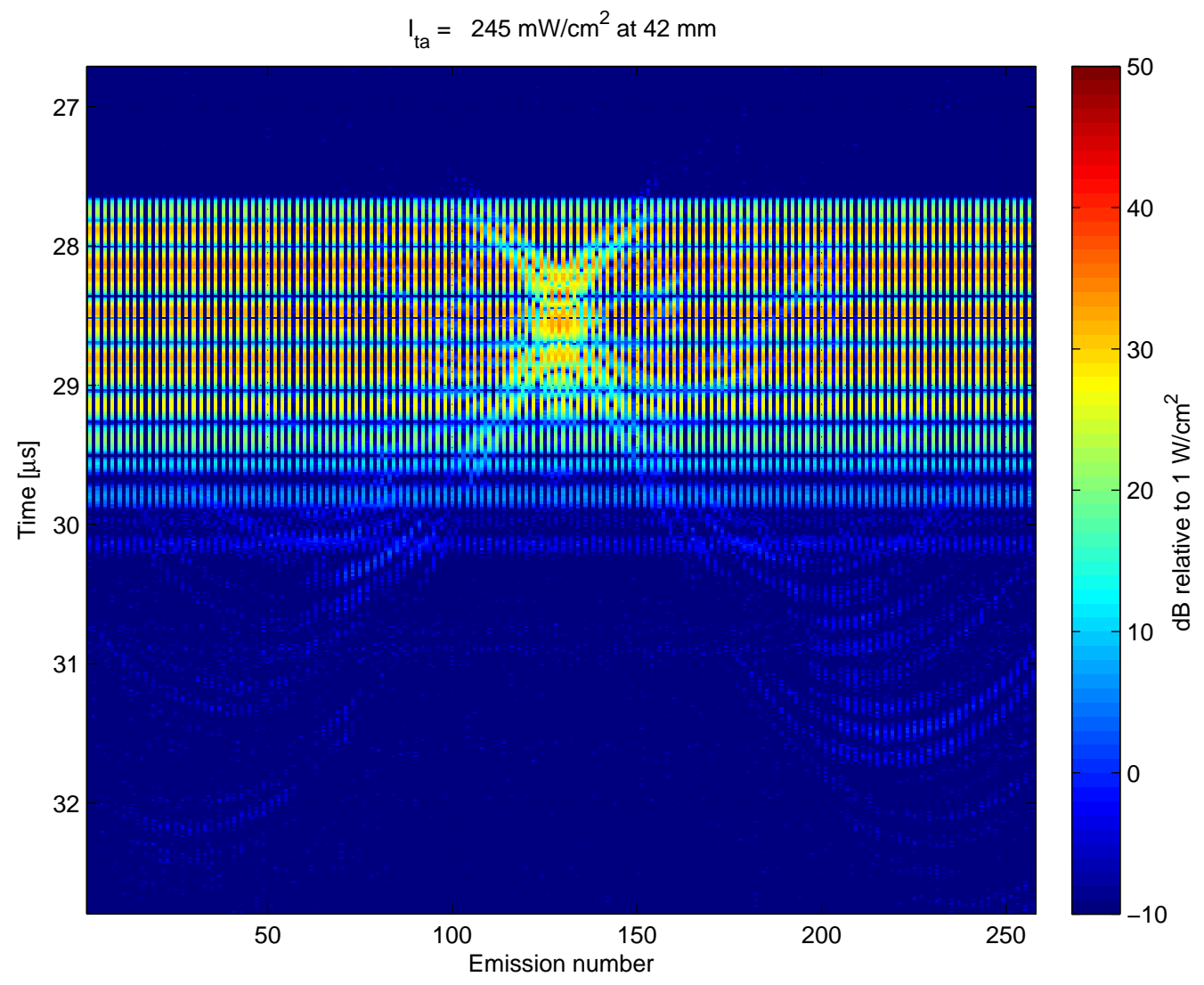

Figure 4. Distribution of the instantaneous intensity as a function of depth and emission number when the hydrophone is $42 \mathrm{~mm}$ from the probe. The color values are shown in $\mathrm{dB}$ relative to $1 \mathrm{~W} / \mathrm{cm}^{2}$.

The distributions of intensities for a number of positions are shown in Fig. 5 for only the B-mode emissions. The B-mode intensity peaks around the elevation focus at $65 \mathrm{~mm}$ and then gradually decrease. The distributions of intensities for a number of positions for the whole sequence are shown in Fig. 6. The general pattern is the same with a significant contribution for every second emission from the flow emission. The flow transmission is focused at $105 \mathrm{~mm}$, and therefore the intensity increases for this until $66 \mathrm{~mm}$, where it starts dropping of. The elevation focus of the array is at $65 \mathrm{~mm}$, and this is the reason for the peak around $66 \mathrm{~mm}$.

The spatial distribution of $I_{t a}$ is shown in Fig. 7. Here the hydrophone has been moved to the spatial position indicated and the duplex sequence has been acquired two times and the signals averaged It peaks around 66 $\mathrm{mm}$ as shown in Fig. 6 with a value of $I_{\text {spta }}=1383 \mathrm{~mW} / \mathrm{cm}^{2}$, which is below the FDA limits of $I_{\text {spta }}=1540$ $\mathrm{mW} / \mathrm{cm}^{2}$. This sequence is, thus, limited to a pulse repetition frequency around $8 \mathrm{kHz}$. It can be seen that the main intensity contribution is placed at the center of the image, since the flow emissions are emitted there. The intensity drops by distance to the acoustic axis and attains a level roughly 15-20 dB below the peak level. This intensity level is due to the B-mode emissions that are spread over a large imaging region. Here the maximum value is also around $65 \mathrm{~mm}$ due to the transducer's elevation focus.

All the $21 \times 101=2121$ measurements were acquired in 8,430 seconds for both emission, data transfer, storage, and motion of the hydrophone. A single measurement, thus, takes 3.97 seconds including the 2.58 seconds for emitting the 258 emissions at $f_{p r f}=100 \mathrm{~Hz}$. The data reading and storage part therefore takes $1.39 \mathrm{~s}$. 

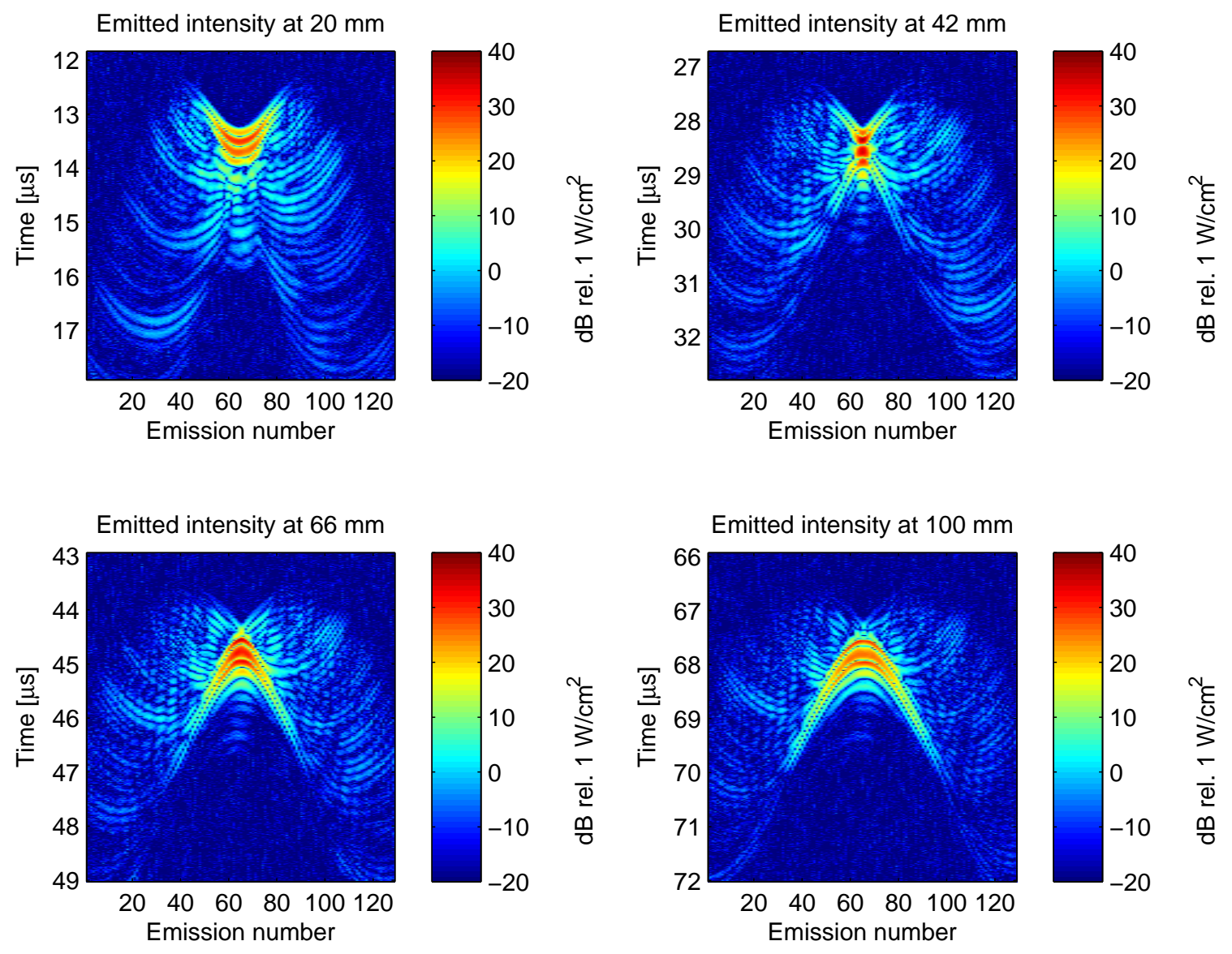

Figure 5. Distribution of the instantaneous intensity as a function of depth and emission number for different depths for the B-mode imaging pulse. The color values are shown in $\mathrm{dB}$ relative to $1 \mathrm{~W} / \mathrm{cm}^{2}$.

\section{DISCUSSION}

The measurement of intensities for complex imaging sequences is complicated. Such sequences often employ a number of different emissions focused in different directions and depths, with various transducer excitations, number of active elements and apodization values. This makes it complicated to predict the place of maximum intensity for the whole sequence. Also the the time between emissions plays a role, and this weights contributions from different emissions.

Traditionally, intensity measurement equipment uses digital oscilloscopes, which are not synchronized to the scanner. They can therefore not detect which emission number currently is measured, and therefore only emission into a single direction must be made. The complex sequence must be split into individual emissions, and the intensity should be measured for each of them. This is a very time consuming process and can also be prone to errors, since many hundred different emissions can be found in one sequence. After measurements the data must be combined to yield the correct spatial peak temporal average intensity with proper weighting of the timing in the sequence. Using a non-synchronized oscilloscope also makes it difficult to average multiple measurements, which either limits the signal-to-noise ratio or introduces further signal processing to align and average the responses.

The approach suggested here is to use the sampling capabilities of modern (research) scanners to acquire all data for a single spatial position. This has several advantages: All pressure signals at one given location are acquired simultaneously and in phase. The signals can readily be averaged over multiple emissions, since they are in phase. The sequence does not have to be taken apart, and it can therefore be ensured that the actual 

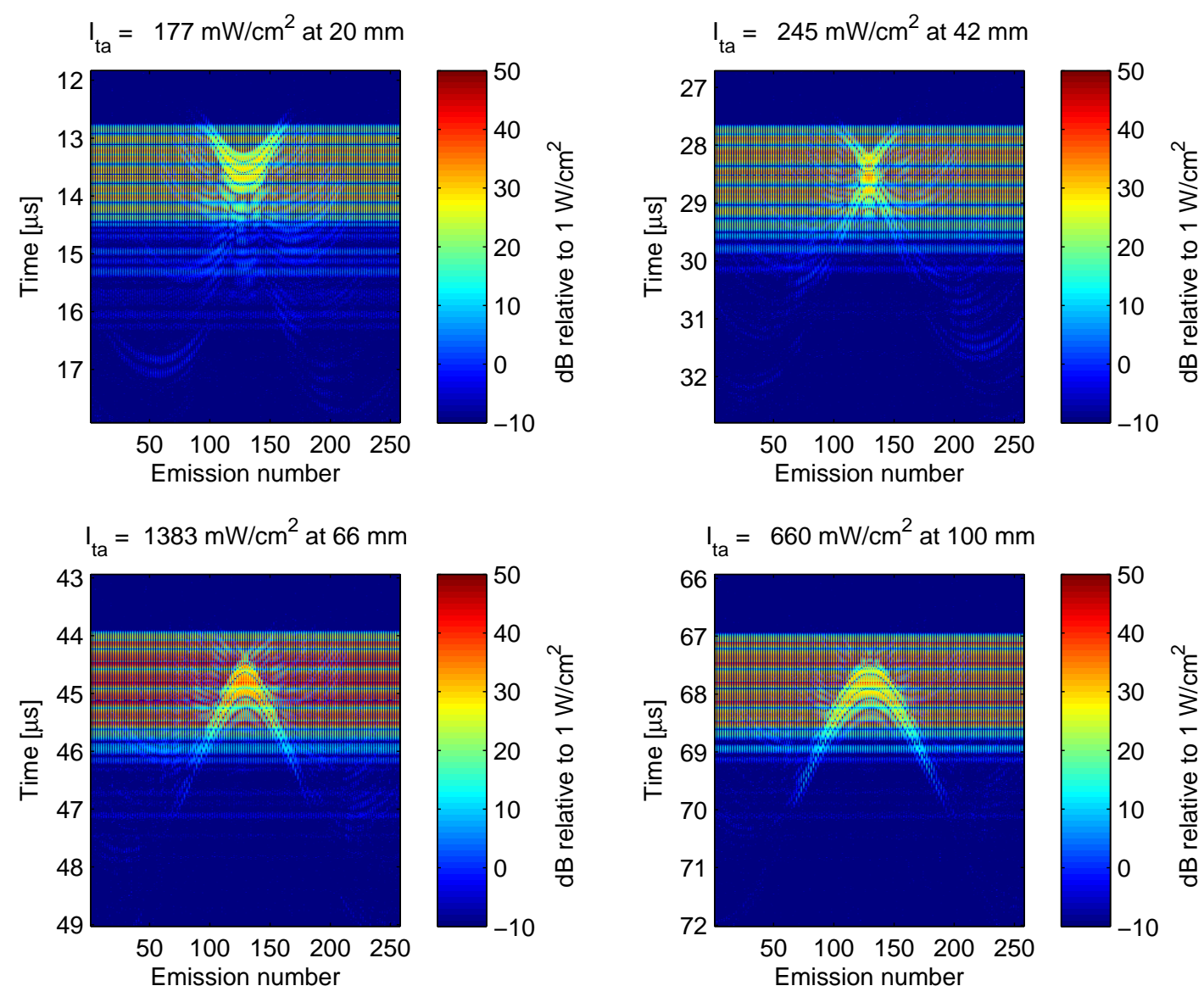

Figure 6. Distribution of the instantaneous intensity as a function of time and emission number for different depths for the duplex imaging sequence. The color values are shown in $\mathrm{dB}$ relative to $1 \mathrm{~W} / \mathrm{cm}^{2}$.

and correct sequence is the one being measured on. These advantages translate into a more reliable and much faster measurement. If the transducer is aligned properly, the measurement only needs to be conducted along the acoustical axis with e.g. 100 positions, and the complete intensity mapping can be conducted in roughly 6 minutes compared to many hours for the traditional approach.

The data presented here for the duplex sequence demonstrates how the spatial peak position is dependent on multiple factors. The $129 \mathrm{~B}$-mode transmissions are focused $42 \mathrm{~mm}$ from the probe surface, use a single cycle excitation, Hanning transmit apodization on the 64 active elements, and are spread out over the full imaging region. The flow emissions are focused at $105 \mathrm{~mm}$, use 4 cycle excitation, the same apodization and the focus is on the acoustical axis. The spatial peak is, however, found at the elevation focus and at the center of the image, but this could change by employing other electronic foci. A measurement of the full sequence is therefore required to reveal the complex intensity map. A shortcut would be to only measure the most dominant emission, which here is the flow emission. The drawback of neglecting the B-mode emission is that this would result in too conservative an intensity measure, and this decreases the available energy and thereby the image quality.

\section{CONCLUSIONS AND RECOMMENDATIONS}

The major advantage of the method presented is that it avoids breaking up an imaging sequence and measuring separate lines in several measurements. This is advantageous in terms of measurement time, and it also ensures that the exact same imaging sequence is used during the measurement. It is therefore possible to make fully generic measurement scripts, which can be directly used and validated. This is a major advantage in a research 


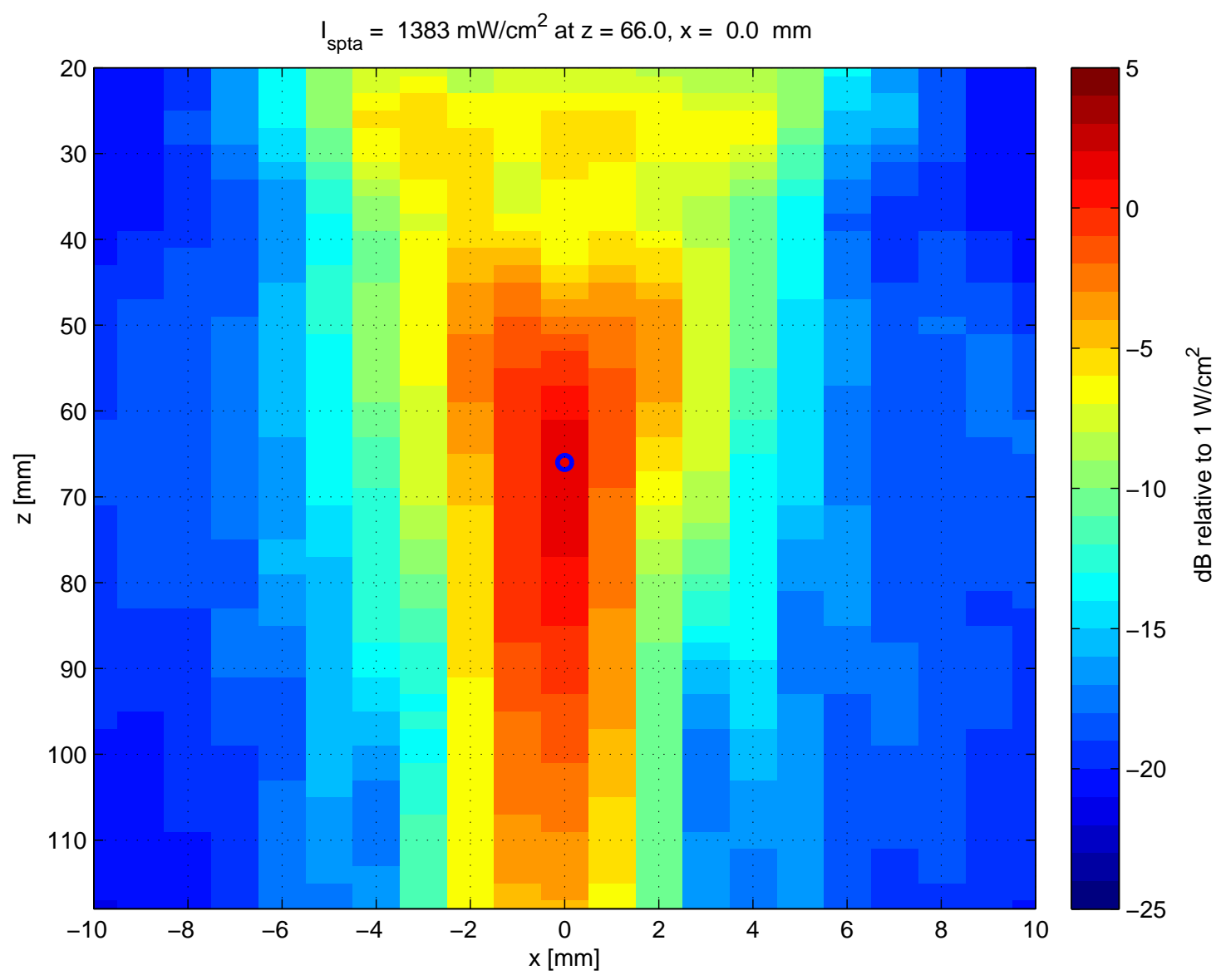

Figure 7. Distribution of $I_{t a}$ as a function of spatial position. The location of the peak intensity $I_{s p t a}$ is shown by the blue circle. The color values are shown in $\mathrm{dB}$ relative to $1 \mathrm{~W} / \mathrm{cm}^{2}$.

environment, where the main focus is not on FDA rules, but on translating new advanced imaging sequences from experiments to clinical trials safely. It can potentially also improve the accuracy and decrease the time spent by ultrasound companies on FDA measurements.

\section{REFERENCES}

[1] FDA, "Information for manufacturers seeking marketing clearance of diagnostic ultrasound systems and transducers," tech. rep., Center for Devices and Radiological Health, United States Food and Drug Administration (1997).

[2] Jensen, J. A., Holm, O., Jensen, L. J., Bendsen, H., Nikolov, S. I., Tomov, B. G., Munk, P., Hansen, M., Salomonsen, K., Hansen, J., Gormsen, K., Pedersen, H. M., and Gammelmark, K. L., "Ultrasound Research Scanner for Real-time Synthetic Aperture Image Acquisition," IEEE Trans. Ultrason., Ferroelec., Freq. Contr. 52 (5), 881-891 (May 2005).

[3] Tortoli, P., Bassi, L., Boni, E., Dallai, A., Guidi, F., and Ricci, S., "ULA-OP: An advanced open platform for ultrasound research," IEEE Transactions on Ultrasonics, Ferroelectrics and Frequency Control 56, 2207-2216 (Oct. 2009).

[4] Jensen, J. A., Holten-Lund, H., Nilsson, R. T., Hansen, M., Larsen, U. D., Domsten, R. P., Tomov, B. G., Stuart, M. B., Nikolov, S. I., Pihl, M. J., Du, Y., Rasmussen, J. H., and Rasmussen, M. F., "SARUS: A synthetic aperture real-time ultrasound system," IEEE Trans. Ultrason., Ferroelec., Freq. Contr. 60(9), 1838-1852 (2013). 
[5] Jensen, J. A. and Svendsen, N. B., "Calculation of Pressure Fields from Arbitrarily Shaped, Apodized, and Excited Ultrasound Transducers," IEEE Trans. Ultrason., Ferroelec., Freq. Contr. 39, 262-267 (1992).

[6] Jensen, J. A., "Field: A program for simulating ultrasound systems," Med. Biol. Eng. Comp. 10th NordicBaltic Conference on Biomedical Imaging, Vol. 4, Supplement 1, Part 1, 351-353 (1996).

[7] Foster, S. G., A pulsed ultrasonic flowmeter employing time domain methods, PhD thesis, Dept. Elec. Eng., University of Illinois, Urbana, Ill. (1985). 